\title{
Flat Panel Angiography in the Cross-Sectional Imaging of the Temporal Bone: Assessment of Image Quality and Radiation Dose Compared with a 64-Section Multisection CT Scanner
}

\author{
(D) G. Conte, DE. Scola, DS. Calloni, (DR. Brambilla, (D) M. Campoleoni, (D). Lombardi, (DF. Di Berardino, DD. Zanetti, (D).M. Gaini,
}

(iD). Triulzi, and (D) C. Sina

\begin{abstract}
BACKGROUND AND PURPOSE: Cross-sectional imaging of the temporal bone is challenging because of the complexity and small dimensions of the anatomic structures. We evaluated the role of flat panel angiography in the cross-sectional imaging of the temporal bone by comparing its image quality and radiation dose with a 64-section multisection CT scanner.
\end{abstract}

MATERIALS AND METHODS: We retrospectively collected 29 multisection CT and 29 flat panel angiography images of normal wholehead temporal bones. Image quality was assessed by 2 neuroradiologists, who rated the visualization of 30 anatomic structures with a 3-point ordinal scale. The radiation dose was assessed with an anthropomorphic phantom.

RESULTS: Flat panel angiography showed better image quality than multisection CT in depicting the anterior and posterior crura of the stapes, the footplate of the stapes, the stapedius muscle, and the anterior ligament of the malleus $(P<.05)$. In contrast, multisection $C T$ showed better image quality than flat panel angiography in assessing the tympanic membrane, the bone marrow of the malleus and incus, the tendon of the tensor tympani, the interscalar septum, and the modiolus of the cochlea $(P<.05)$. Flat panel angiography had a significantly higher overall image quality rating than multisection $\mathrm{CT}(P=.035)$. A reduction of the effective dose of approximately $40 \%$ was demonstrated for flat panel angiography compared with multisection CT.

CONCLUSIONS: Flat panel angiography shows strengths and weaknesses compared with multisection CT. It is more susceptible to artifacts, but due to the higher spatial resolution, it shows equal or higher image quality in assessing some bony structures of diagnostic interest. The lower radiation dose is an additional advantage of flat panel angiography.

ABBREVIATIONS: FPA = flat panel angiography; $\mathrm{FPCT}=$ flat panel $\mathrm{CT} ; \mathrm{H}_{\mathrm{T}}=$ equivalent dose; $\mathrm{MSCT}=$ multisection $\mathrm{CT} ; \mathrm{TLD}=$ thermoluminescent dosimeter

$\mathrm{C}_{\mathrm{b}}$ ross-sectional imaging of the temporal bone is challenging because of the complexity and small dimensions of the anatomic structures. Multisection CT (MSCT) represents the technique of choice for the study of the temporal bones. It is noninvasive and provides a high spatial resolution $(\sim 0.4 \mathrm{~mm}$ in-plane and $\sim 0.5 \mathrm{~mm}$ in section thickness) that allows radiologists to

Received January 17, 2017; accepted after revision May 22.

From the Postgraduation School of Radiodiagnostics (G.C., S.C.) and Department of Pathophysiology and Transplantation (F.T.), Università degli Studi di Milano, Milan, Italy; Neuroradiology Unit (E.S., L.L., F.T., C.S.) and Health Physics Unit (R.B., M.C.), Fondazione Istituto Di Ricovero e Cura a Carattere Scientifico Ca'Granda Ospedale Maggiore Policlinico, Milan, Italy; and Audiology Unit (F.D.B., D.Z.) and Otolaryngology Unit (L.M.G.), Department of Clinical Sciences and Community Health, Fondazione Istituto Di Ricovero e Cura a Carattere Scientifico Ca'Granda Ospedale Maggiore Policlinico, Università degli Studi di Milano, Milan, Italy.

Please address correspondence to Giorgio Conte, MD, Postgraduation School in Radiodiagnostics, Università degli Studi di Milano, Via Festa del Perdono 7, 20122 Milan, Italy; e-mail: giorgioconte.unimed@gmail.com

三 Indicates article with supplemental on-line tables.

Indicates article with supplemental on-line photos.

http://dx.doi.org/10.3174/ajnr.A5302 visualize most of the anatomic structures and detect pathologic changes. ${ }^{1}$ On occasion, however, more invasive examinations, such as an exploratory operation, may be required to achieve a diagnosis or to clarify the pathologic processes depicted by MSCT. $^{2}$

Flat panel CT (FPCT) has recently provided an alternative method with ultra-high isotropic spatial resolution $(\sim 150 \times$ $\left.150 \times 150 \mu \mathrm{m}^{3}\right)^{3,4}$ Some studies have investigated the image quality of FPCT in the delineation of postmortem normal temporal bone anatomy: FPCT showed higher image quality compared with MSCT when isolated temporal specimens were scanned but showed similar image quality in cadaveric whole-head specimens. ${ }^{1,2,3,5}$ The diagnostic value of FPCT has been reported to be high in the assessment of conductive hearing loss. ${ }^{6}$

All these studies have been using prototype scanners or dedicated scanners to perform FPCT of the temporal bone. Radiologic assessment of the fine bony structures of the ear can also be performed with angiographic systems equipped with flat panel detectors, but the use of this technique is still uncommon. Recently, flat 
panel angiography (FPA) was used in the cross-sectional imaging assessment of cochlear implants and metallic prostheses after middle ear reconstructive surgery because it is less susceptible to metallic artifacts. ${ }^{7-9}$ However, the image quality of FPA of the normal temporal bone has not yet been investigated in patients during clinical practice, and radiation exposure has not been assessed so far, to our knowledge. It was our expectation that FPA could provide better image quality at lower radiation exposure compared with MSCT.

The purpose of our study was to test the value of FPA in the cross-sectional imaging of the temporal bone in a cohort of patients during clinical practice by comparing its image quality and radiation dose with those in 64-section MSCT.

\section{MATERIALS AND METHODS \\ Patients}

The use of FPA in our department began in January 2015 for the postoperative assessment of patients with cochlear implants, ${ }^{7}$ after its radiation exposure was tested on a phantom. Thereafter, the use of FPA was extended to other clinical indications on the basis of its proved image quality. FPA and MSCT were randomly performed. FPA was always preferred to MSCT because of the results of the phantom dose experiments (see the "Radiation Dose Assessment" paragraph in the "Results" section) but was performed whenever the unit was available, the latter being largely dedicated to endovascular procedures. Otherwise MSCT was performed.

For this study, from January 2015, we collected 29 consecutive MSCT scans and 29 consecutive FPA scans of normal temporal bones, obtained in our department. Temporal bones were defined as normal according to the following criteria: 1) no pathologic findings on MSCT or FPA, and 2) no history of otologic disorders on the imaged side as evidenced by the patient's medical record. Each examination was performed to investigate or rule out suspected abnormalities in the contralateral temporal bone, including cholesteatoma, otosclerosis, middle and inner ear congenital malformations, and complications of otitis.

This retrospective study received review board approval; patient informed consent was waived.

\section{MSCT and FPA Protocols}

MSCT examinations were performed with a 64-section CT scanner (Optima CT660; GE Healthcare, Milwaukee, Wisconsin). The MSCT scan included both temporal bones, with the following scan parameters: current, $200 \mathrm{~mA}$; voltage, $120 \mathrm{kV}$; pitch, 0.531:1; rotation time, 1 second; section collimation, $0.625 \mathrm{~mm}$; FOV, 22 $\mathrm{cm}^{2}$; matrix, $512 \times 512$; scan length, $105 \mathrm{~mm}$. The scan time was 5.63 seconds. The CT dose index volume was $62.1 \mathrm{mGy}$, and the dose-length was $652 \mathrm{mGy} \times \mathrm{cm}$. The images were reconstructed from the raw data with a $512 \times 512$ matrix and a $10-\mathrm{cm}$ FOV, leading to a $0.195 \times 0.195 \mathrm{~mm}$ pixel size in the plane of acquisition. A bone sharpening filter (Bone Plus; GE Healthcare) was used.

FPA was performed with an angiographic system (Allura Xper FD20; Philips Healthcare, Best, the Netherlands), including a digital flat panel detector, $30 \times 40 \mathrm{~cm}$ with a source-to-image-receptor distance of $120 \mathrm{~cm}$. The FPA scan included both temporal bones of the patient's head, with the following scan parameters: current, $260 \mathrm{~mA}$; voltage, $80 \mathrm{kV}$; FOV, $20 \times 15 \mathrm{~cm}^{2}$; voxel size, $0.14 \times 0.14 \times 0.14 \mathrm{~mm}^{3}$; scan height, $150 \mathrm{~mm}$. By rotating $240^{\circ}$ (from $60^{\circ}$ to $300^{\circ}$ ) passing through the posterior part of the head and avoiding the anterior part, the pivoting $\mathrm{C}$-arm of the angiography unit acquires a volume dataset of up to 622 projections, with a scan time of 25 seconds. The dose-area product was 10,650 $\mathrm{mGy} \times \mathrm{cm}^{2}$, and the air kerma was $121 \mathrm{mGy}$. Each temporal bone was reprocessed separately into a small FOV. Postprocessing of this volume dataset was performed with reconstruction software (Allura 3D-RA 6.3.0/XperCt 3.1.0; Philips Healthcare), offering all the possibilities of standard 3D-postprocessing such as multiplanar reformations, curved reformations, volume-rendering technique, shaded surface display technique, and MIP. Depending on the number of additional procedures running, the average reconstruction time was approximately 10 minutes.

\section{Assessment of Image Quality}

Two neuroradiologists with $>3$ years of experience in otoradiology independently evaluated each examination separately on a PACS viewer. The readers were permitted to scroll through the image sections, change the CT window level and width, perform MPR and MIP of the volume data, and zoom in and out in any order.

First, the radiologists defined each scan as adequate or inadequate according to the presence of artifacts, including head movements. Thirty anatomic structures were identified, listed in Online Table 1. For each of the structures, the investigators rated the quality of visualization with an ordinal scale as follows: Zero indicated that the anatomic structure could not be identified; 1 , the anatomic structure could be identified but was not well-delineated from the surrounding structures; and 2, the anatomic structure could be identified and was well-delineated from the surrounding structures. For each examination, the summed score represented the overall image quality, ranging from 0 to 60 points (maximum, 2 points $\times 30$ structures). The 2 readers' average scores (continuous variables) were used to compare differences between MSCT and FPA.

Furthermore, each reader placed a circular ROI (approximately $50 \mathrm{~mm}^{2}$ ) in the inner portion of the external acoustic canal, avoiding surrounding bony structures and the tympanic membrane. The signal was defined as the mean CT attenuation value within the ROI, and the noise, as the SD of the CT attenuation values within the ROI. The signal-to-noise ratio was calculated. The 2 readers' average signal, noise, and SNR were used to compare MSCT and FPA.

Nominal variables were represented as relative percentages, and continuous variables were represented as the mean \pm SD. A $\chi^{2}$ test for nominal data and a Mann-Whitney test for continuous data were used to compare MSCT and FPA. The KolmogorovSmirnov test was used to test the normality of the continuous variables, and an unpaired Student $t$ test or Mann-Whitney test was used to compare the 2 groups, as appropriate. The Spearman rank correlation coefficient was used to assess the degree of correlation between SNR and the summed score for each group. To analyze the interobserver agreement, we dichotomized into 2 
groups ("visualized" = 1 ; and "not visualized" =0). The observers' sensitivity in detecting each anatomic structure was calculated, and interobserver agreement was expressed as a percentage of agreement. The $\kappa$ statistic, which estimates the proportion of interobserver agreement above that expected by chance, was computed for each anatomic structure. Differences were considered statistically significant with $P<.05$. The statistical analysis was performed with SPSS 20 statistical software (IBM, Armonk, New York).

\section{Radiation Dose Assessment}

We used an anthropomorphic Rando Alderson phantom (Rando Alderson Research Laboratories, Long Island, City, New York) for radiation dose assessment. The phantom represented an average male and was composed of a human skeleton embedded in tissueequivalent material. A thermoluminescent dosimeter (TLD) (GR200 Thermoluminescent Detector; 360RAD, Beijing, China) consisting of $\mathrm{LiF}, \mathrm{Mg}, \mathrm{Cu}$, and $\mathrm{P}$ materials (https://www.thermofisher.com/ order/catalog/product/SCP18815) with an intrinsic dose detector limit of approximately 1 microsievert ( $\mu \mathrm{Sv}$ ) was used. The TLDs were inserted into the Rando phantom at sites corresponding to organs or tissues (listed in On-line Table 2) of interest in the head and neck region. ${ }^{10}$ In addition, we placed TLDs outside on the phantom orbits to estimate the dose on the eye lenses. Four TLDs were placed at each site to obtain the average, the SD, and the variation coefficient. For each technique (MSCT and FPA), 3 scans were obtained to provide a more reliable measure of radiation in the dosimeters, minimizing the changes related to the phantom positioning.

Estimation percentages of irradiated tissue were retrieved from the literature and used to calculate the equivalent dose $\left(H_{\mathrm{T}}\right)$ to a tissue or organ in $\mu$ Sv. ${ }^{10,11}$ Effective dose $(E)$, expressed in $\mu \mathrm{Sv}$, was calculated with the following equation: $E=\Sigma w T \times H_{\mathrm{T}}$, where $E$ is the product of the tissue-weighting factor $(w T)$, which represents the relative contribution of that organ or tissue to the overall risk, and the $H_{\mathrm{T}}$. The whole body was found by the summation of the weighted equivalent doses to all tissues or organs exposed. The International Commission on Radiologic Protection 103/2007 tissue-weighting factors were used to calculate $E .^{12}$

\section{RESULTS}

\section{Image Quality Assessment}

All 29 MSCT and FPA scans were considered adequate by both readers. In particular, no FPA scan showed excessive head movement, necessitating the repetition of the examination. The MSCT and FPA groups did not differ in age $(49.0 \pm 6.9$ versus $46.8 \pm$ 12.5 years; $P=.466$ ), sex (female/male: $19: 10$ versus $18: 11 ; P=$ .785 ), or side (right/left: $16: 13$ versus $15: 14 ; P=.792)$. MSCT had higher signal $(938.4 \pm 21.0$ versus $767.0 \pm 86.8 ; P<.001)$, lower noise $(61.5 \pm 14.4$ versus $236.2 \pm 44.0 ; P<.001)$, and higher SNR $(16.39 \pm 3.8$ versus $3.5 \pm 1.1 ; P<.001)$ compared with FPA.

MSCT showed better image quality than FPA in assessing the tympanic membrane, bone marrow of the malleus and incus, tendon of tensor tympani, interscalar septum and modiolus of the cochlea (all statistics, $P<.05$; On-line Table 1 and On-line Fig 1 ). FPA showed better image quality than MSCT in assessing the anterior and posterior crura of the stapes (On-line Fig 2), footplate of the stapes (On-line Fig 2), incudostapedial joint (On-line
Table 1: Equivalent doses $\left(H_{T}\right)$ for each organ of interest

\begin{tabular}{lcc}
\multicolumn{1}{c}{ Sites } & FPA & MSCT \\
\hline Bone marrow & 2.4 & 3.24 \\
Esophagus & 0.15 & 0.37 \\
Thyroid & 1.48 & 3.74 \\
Skin & 0.33 & 2.09 \\
Bone surface & 6.05 & 6.89 \\
Salivary glands & 32.44 & 57.26 \\
Brain & 21.33 & 32.71 \\
Lymphatic nodes & 0.69 & 1.15 \\
Muscle & 0.69 & 1.15 \\
Extrathoracic region & 12.93 & 26.6 \\
Oral mucosa & 15.71 & 25.21 \\
Pituitary & 24.31 & 45.84 \\
Eyes & 5.09 & 51.15 \\
Eye lens & 1.77 & 45.9 \\
\hline
\end{tabular}

Table 2: Tissue-weighting factors and effective doses of FPA and MSCT for each organ

\begin{tabular}{lccc}
\hline \multicolumn{1}{c}{ Sites } & $\begin{array}{c}\text { Tissue-Weighting } \\
\text { Factors (ICRP } \\
\text { 103 Version) }\end{array}$ & FPA & MSCT \\
\hline Bone marrow & 0.12 & 0.29 & 0.39 \\
Esophagus & 0.04 & 0.01 & 0.01 \\
Thyroid & 0.04 & 0.06 & 0.15 \\
Skin & 0.01 & 0.00 & 0.02 \\
Bone surface & 0.01 & 0.06 & 0.07 \\
Salivary glands & 0.01 & 0.32 & 0.57 \\
Brain & 0.01 & 0.21 & 0.33 \\
Lymphatic nodes & & & \\
Muscle & 0.12 & 0.28 & 0.5 \\
Extrathoracic region & & & \\
Oral mucosa & Mean dose on remainder organs & \\
Effective dose (mSv) & \multicolumn{3}{c}{1.23} \\
\hline
\end{tabular}

Note:-ICRP indicates International Commission on Radiological Protection.

${ }^{a}$ Remainder organs are: adrenals, extrathoracic region, gall bladder, heart, kidneys, lymphatic nodes, muscle, oral mucosa, pancreas, prostate or uterus/cervix, small intestine, spleen, thymus. For remainder organs a tissue-weighting factor of 0.05 is assigned according to the IRCP 103 version. The effective dose for the extrathoracic region and the oral mucosa, respectively, corresponds to the mean dose on remainder organs.

Fig 3), and stapedius muscle and anterior ligament of the malleus (all statistics, $P<.05$; On-line Table 1). No significant difference in terms of image quality was found between MSCT and FPA in the assessment of the remaining anatomic structures (On-line Table 1). FPA had a significantly higher overall image quality (summed score) than MSCT ( $P=.035$; On-line Table 1$)$. Data on the observer sensitivity and interobserver agreement are summarized in On-line Table 3. The summed score of FPA correlated with the $\operatorname{SNR}(R=0.44, P=.02)$, while no correlation was found between the summed scores of MSCT and SNR $(R=0.21, P=.26)$.

\section{Radiation Dose Assessment}

Doses of the TLD sites are reported in On-line Table 2 and represented in On-line Fig 4; equivalent doses and effective doses for each organ are reported in Tables 1 and 2, respectively.

\section{DISCUSSION}

\section{Image Quality Assessment}

Our study demonstrates that FPA provides higher image quality in the cross-sectional imaging of the temporal bone compared with a 64-section MSCT scanner. To the best of our knowledge, 
no previous studies have assessed the image quality of FPA in delineating normal temporal bone anatomy.

The image quality of FPCT prototypes or dedicated FPCT scanners has already been assessed in isolated temporal bone specimens, resulting in better image quality compared with MSCT. ${ }^{1,3,13}$ However, these results are not clinically useful because FPCT prototypes are not available for clinical use in humans; the use of dedicated FPCT scanners in isolated temporal bone specimens does not represent the real clinical setting. Whole-head specimens absorb more low-energy photons, and radiation penetration is lower because the mass between the radiation source and the detector is greater.

A previous study showed that a dedicated FPCT scanner did not provide improvement in image quality in scanning wholehead temporal bone specimens compared with MSCT. ${ }^{1}$ Our results are partially in agreement with these results. Although the overall image quality of FPA was better than MSCT, FPA improved the visualization of some structures (ie, stapes, stapedius muscle, anterior malleus ligament, chorda tympani), but it showed a limited value in the visualization of other structures (ie, modiolus, the interscalar septum of the cochlea, the tympanic membrane, bone marrow of the malleus and incus). Some technical aspects are pertinent to understanding these heterogeneous results.

The high isotropic spatial resolution of FPA $(0.14 \times 0.14 \times$ $0.14 \mathrm{~mm}$ ) provided more detailed cross-sectional images and allowed us to obtain multiplanar reformations with the same spatial resolution as in the original plane. In contrast, MSCT had a lower spatial resolution $(0.64-\mathrm{mm}$ thickness and $0.2 \times 0.2 \mathrm{~mm}$ in-plane resolution), and the anisotropic voxel shape led to a loss of detail in the MPR images. The higher spatial resolution of FPA was particularly useful for the depiction of small bone structures such as the stapes and its components but was less useful for the visualization of larger bone structures, such as the malleus and incus, which were well-visualized by MSCT.

FPA had different technical disadvantages compared with MSCT, including a longer acquisition time, more susceptibility to artifacts, and lower contrast resolution and SNR. The longer acquisition time of FPA ( 25 versus 5.6 seconds) can render the scanning more susceptible to motion artifacts. Beam-hardening and scattering artifacts were evident in FPA, creating bright streaks in the image. Beam-hardening occurs in high-attenuation tissue, such as the temporal bone, where low-energy photons are more easily absorbed and high-energy photons pass through. ${ }^{14}$ Thus, beam transmission does not follow the simple exponential decay seen with a monochromatic $x$-ray. FPA cannot use the same algorithms that MSCT adopts to correct for beam-hardening because they require that the complete object (ie, the temporal bone) fit into the FOV; FPA uses a small FOV $(20 \times 25 \mathrm{~cm})$ and does not completely compute the temporal bone. ${ }^{15}$

Scattering artifacts are due to the deviation of some photons when they pass through the whole-head tissues. The diverted photons impact the wrong detector (ie, not the detector parallel to the $\mathrm{X}$-ray source), and tissue density information is distorted. ${ }^{1}$ In FPA, unlike MSCT, scatter intensities may severely impair image quality because the entire volume, as opposed to a few planes in MSCT, contributes to the scattered radiation. ${ }^{4}$ Beam-hardening and scattering artifacts explain why FPA visualized small bone structures surrounded by soft-tissue (ie, the modiolus and interscalar septum of the cochlea) or small soft-tissue structures surrounded by bony structures (ie, bone marrow of the malleus and incus) less optimally than MSCT. In addition, scattering artifacts were responsible for the low contrast resolution of FPA, which did not attain improved depiction of small soft-tissue structures immersed in air space (ie, suspensory ligaments, tendons of the tensor tympani and tympanic membrane) compared with MSCT, as one could expect. The high susceptibility to artifacts explains the higher image noise and the lower signal and SNR measured in the FPA images. We demonstrated that when artifacts decreased and SNR increased, the overall image quality of FPA improved.

Considering our results, it is important to define when the use of FPA is preferable to MSCT for the cross-sectional imaging of the temporal bone. FPA is recommended only in patients with high compliance to avoid head motion artifacts due to the longer acquisition time. We always preferred FPA in the assessment of the middle and inner ear because it shows equal or better image quality than MSCT in depicting almost all bony structures of diagnostic interest. Although FPA could fail in the assessment of the modiolus and the interscalar septum because of motion, beamhardening, and scattering artifacts, we also recommend FPA for the assessment of suspected cochlear malformations in pediatric patients. In fact, beam-hardening and scattering artifacts are usually less evident in pediatric patients due to their smaller head volume. In uncooperative pediatric patients, we perform FPA with the patient under sedation in the same session with the MR imaging study, if planned, to avoid 2 separate sedations. Otherwise MSCT with low-dose protocol is preferred. FPA is highly recommended in the assessment of cochlear implants and metallic prostheses after middle ear reconstructive surgery, as previously reported in the literature. ${ }^{7-9}$ Regarding the cross-sectional imaging of the outer ear, we use FPA for the assessment of the bony external auditory canal, as in cases of suspected external auditory canal atresia or osteoma, which may be associated with middle ear malformations. In contrast, we do not use FPA for the assessment of pathologies that can involve the soft tissues of the periauricular region, such as malignant otitis externa because of the low contrast resolution of FPA.

\section{Radiation Dose Assessment}

According to our results, the lower radiation dose is the main advantage of FPA in the assessment of the temporal bone. Compared with MSCT, FPA showed a reduction of the effective dose of up to $40 \%$ ( 1.23 versus $2.04 \mathrm{mSv}$ ). We observed that the equivalent dose of FPA decreased approximately $76 \%$ for the lenses of the eye and $40 \%$ for the thyroid, which are the most radiosensitive tissues in the head and neck region.

To the best of our knowledge, no previous studies have compared radiation doses between FPCT or FPA and MSCT in the assessment of the temporal bone. In the literature, similar data were only reported for FPCT in the assessment of the maxillofacial region, reflecting the widespread use of FPCT in dental radiology. ${ }^{10,16-19}$ In previous studies considering radiation exposure of FPCT and MSCT in the assessment of the temporal bone, the dose 
was provided by the manufacturers ${ }^{2}$ or recorded from the scanner console. $^{13}$

The lower equivalent dose of FPA is due to 3 main reasons: The first is related to the scan parameters because the dose change is approximately proportional to the square of the tube voltage (kilovolt) and proportional to the current (milliampere). ${ }^{20}$ Thus, the lower tube voltage of FPA determines a reduction of the dose though its current is higher. Second, FPA has a smaller FOV than MSCT, allowing a further reduction in exposure as well as scanning of the whole mastoid area together with the middle and inner ears, which may be essential for some diagnoses. ${ }^{13}$ Third, the semicircular trajectory of our x-ray source, posterior to the head, explains the conspicuous reduction in radiation dose to the lenses of the eyes and the thyroid. Regions situated in the primary beam of the $\mathrm{x}$-ray source receive a higher dose than regions far from the source.

Some limitations of our study must be acknowledged. We did not perform a within-subject analysis, with each patient undergoing both examinations, for ethical reasons; however, this limitation was overcome by enrolling a large number of subjects. We do not know whether our results will be completely reproducible with other angiography systems because of the different technology and reconstruction algorithms provided by various manufacturers. Our image quality assessment was focused on the delineation of the normal ear anatomy, so we do not know if the described improvements of FPA can improve the diagnosis of pathologic processes. Finally, we did not compare the FPA system and FPCT scanner, so we do not know if the latter remains the first choice for the temporal bone assessment.

\section{CONCLUSIONS}

FPA can be adopted for the cross-sectional imaging of the temporal bone, showing strengths and weaknesses compared with MSCT. FPA can fail in depicting structures, as discussed in this article, because of motion, beam-hardening, and scattering artifacts that impair the contrast resolution. However, due to its higher spatial resolution, FPA showed equal or higher image quality than MSCT in assessing bony structures of considerable diagnostic interest for radiologists. The lower radiation dose is an additional advantage of FPA, showing a reduction of the effective dose of up to $40 \%$ compared with MSCT.

\section{REFERENCES}

1. Fatterpekar GM, Doshi AH, Dugar M, et al. Role of 3D CT in the evaluation of the temporal bone. Radiographics 2006;26(suppl 1): S117-32 CrossRef Medline

2. Majdani O, Thews K, Bartling S, et al. Temporal bone imaging: comparison of flat panel volume CT and multisection CT. AJNR Am J Neuroradiol 2009;30:1419-24 CrossRef Medline

3. Gupta R, Bartling SH, Basu SK, et al. Experimental flat-panel high- spatial-resolution volume CT of the temporal bone. AJNR Am J Neuroradiol 2004;25:1417-24 Medline

4. Gupta R, Cheung AC, Bartling SH, et al. Flat-panel volume CT: fundamental principles, technology, and applications. Radiographics 2008;28:2009-22 CrossRef Medline

5. Dalchow CA, Weber Al, Yanagihara $\mathrm{N}$, et al. Digital volume tomography: radiologic examinations of the temporal bone. AJR Am J Roentgenol 2006;186:416-23 CrossRef Medline

6. Dalchow CV, Weber AL, Bien S, et al. Value of digital volume tomography in patients with conductive hearing loss. Eur Arch Otorhinolaryngol 2006;263:93-99 CrossRef Medline

7. Pearl MS, Roy A, Limb CJ. High-resolution secondary reconstructions with the use of flat panel CT in the clinical assessment of patients with cochlear implants. AJNR Am J Neuroradiol 2014;35: 1202-08 CrossRef Medline

8. Zaoui K, Kromeier J, Neudert M, et al. Flat panel CT following stapes prosthesis insertion: an experimental and clinical study. Eur Radiol 2012;22:837-44 CrossRef Medline

9. Zaoui K, Kromeier J, Neudert M, et al. Clinical investigation of flat panel CT following middle ear reconstruction: a study of 107 patients. Eur Radiol 2014;24:587-94 CrossRef Medline

10. Ludlow JB, Ivanovic M. Comparative dosimetry of dental CBCT devices and 64-slice CT for oral and maxillofacial radiology. Oral Surg Oral Med Oral Pathol Oral Radiol Endod 2008;106:106-14 CrossRef Medline

11. Kim DS, Rashsuren O, Kim EK. Conversion coefficients for the estimation of effective dose in cone-beam CT. Imaging Sci Dent 2014; 44:21-29 CrossRef Medline

12. The 2007 Recommendations of the International Commission on Radiological Protection: ICRP Publication 103. Ann ICRP 2007;37: 1-332 CrossRef Medline

13. Peltonen LI, Aarnisalo AA, Kortesniemi MK, et al. Limited conebeam computed tomography imaging of the middle ear: a comparison with multislice helical computed tomography. Acta Radiol 2007;48:207-12 CrossRef Medline

14. Brooks RA, Di Chiro G. Beam hardening in $\mathrm{x}$-ray reconstructive tomography. Phys Med Biol 1976;21:390-98 CrossRef Medline

15. Schlicht FA, Kunze H. Beam hardening correction for flat-panel computed tomography devices. In: Proceedings of the International Meeting on Fully-Dimensional Image Reconstruction in Radiology and Nuclear Medicine, Newport, Rhode Island. June 1-4, 2015

16. Cohen $\mathrm{M}$, Kemper J, Mobes $\mathrm{O}$, et al. Radiation dose in dental radiology. Eur Rad 2002;12:634-37 CrossRef Medline

17. Li G. Patient radiation dose and protection from cone-beam computed tomography. Imaging Sci Dent 2013;43:63-69 CrossRef Medline

18. Loubele M, Bogaerts R, Van Dijck E, et al. Comparison between effective radiation dose of CBCT and MSCT scanners for dentomaxillofacial applications. Eur J Radiol 2009;71:461-68 CrossRef Medline

19. Veldhoen S, Schollchen M, Hanken H, et al. Performance of conebeam computed tomography and multidetector computed tomography in diagnostic imaging of the midface: a comparative study on phantom and cadaver scans. Eur Rad 2017;27:790-800 CrossRef Medline

20. Kaira MK, Maher MM, Toth TL, et al. Strategies for CT radiation dose optimization. Radiology 2004;230:619-28 CrossRef Medline 